\title{
2-乙酰氧甲基吡咯衍生物和醇钠的醚化反应研究
}

\author{
严兆华* 余章昕 刘永杰 胡 伟 \\ (南昌大学理学院化学系 南昌 330031)
}

\begin{abstract}
摘要 研究了 2-乙酰氧甲基吡咯衍生物和醇钠在回流条件下的醚化反应，结果以优秀的收率生成了相应的 2-烷氧甲基 吡咯衍生物. 提出了一个基于氮杂富勒烯过渡态的反应机理用于解释实验结果，提供了一种在碱性介质中制备 2-烷氧 甲基吡咯衍生物的新方法.
\end{abstract}

关键词 2-乙酰氧甲基吡咯衍生物; 2-烷氧甲基吡咯衍生物; 醚化反应; 氮杂富勒烯; 醇钠

\section{Etherification of 2-Acetoxymethylpyrrole Derivative with Sodium Alkoxide}

\author{
Yan, Zhaohua* $\quad$ Yu, Zhangxin $\quad$ Liu, Yongjie $\quad \mathrm{Hu}$, Wei \\ (Department of Chemistry, Nanchang University, Nanchang 330031)
}

\begin{abstract}
Etherification of 2-acetoxymethylpyrrole derivative with sodium alkoxide at reflux was investigated in details resulting in the smooth formation of the corresponding 2-alkoxymethylpyrrole derivative in excellent yield. A plausible mechanism involving the formation of a highly reactive intermediate azafulvene species was proposed to explain the observed transformation. An alternate method for the preparation of 2-alkoxymethylpyrrole derivative starting from 2-acetoxymethylpyrrole derivative in alkaline media was provided.
\end{abstract}

Keywords 2-acetoxymethylpyrrole derivative; 2-alkoxymethylpyrrole derivative; etherification; azafulvene; sodium alkoxide

2-烷氧甲基吡咯衍生物因其比 2-羊全甲基吡咯衍生物 和 2-乙酰氧甲基吡咯衍生物具有更高的稳定性和更好 的反应选择性而可被用于制备低聚吡咯衍生物、卟啉衍 生物、杯吡咯和其它一些具有生理活性的含吡咯杂环化 合物中 ${ }^{[1,2]}$. 近年来, 随着对吡咯衍生物的进一步研究, 人们又发现了吡咯衍生物在新型材料化学领域的一些 新的用途 ${ }^{[3]}$. 吡咯衍生物的新方法学研究自然就引起 了人们浓厚的兴趣. 文献上至今未见系统地考察 2-烷氧 甲基吡咯衍生物的制备方法, 仅仅是零星地在几篇文献 中报道过了 $N$-取代的 2-羊圣甲基吡咯衍生物和卤代物在 氢化钠作用下的醚化反应 ${ }^{[6]} 、 2$-乙酰氧甲基吡咯衍生物 在稀盐酸催化下于回流中与醇的反应 ${ }^{[7]}$ 和 2-乙酰氧甲基

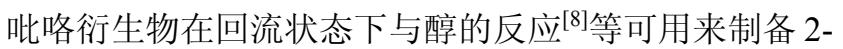
烷氧甲基吡咯衍生物. 有些 2-羟甲基吡咯衍生物或 2-乙 酰氧甲基吡咯衍生物在酸性和高温条件下很容易聚合
或者不稳定易于分解，因此现有的制备 2-烷氧甲基吡咯 衍生物的方法存在着产物复杂和产率低等缺点. 我们最 近正在研究 2-烷氧甲基吡咯衍生物和吡咯-2-甲醛缩醛 衍生物在一些路易斯酸催化下的新型模式聚合反应以 获得新型结构的杂环化合物用于生理活性的测试, 因此 需要制备一系列的 2-烷氧甲基吡咯衍生物和吡咯-2-甲 醛缩醛衍生物底物用于考察反应. 在研究中我们已经发 现了基于高度活泼的 “氮杂富勒烯(Azafulvene)” 中间体 形成机理的吡咯-2-甲醛缩醛衍生物和醇钠在醇中的缩 醛交换反应可以很方便和高效地合成一系列所需要的 吡咯-2-甲醛缩醛衍生物 ${ }^{[9]}$. 在此基础上, 本文继续报道 一种方便而高效的制备 2-烷氧甲基吡咯衍生物的新方 法，即 2-乙酰氧甲基吡咯衍生物和醇钠于回流状态下在 醇或四氢呋喃中能发生醚化反应，结果以优秀的收率生 成了相应的 2-烷氧甲基吡咯衍生物，见 Eq. 1.

\footnotetext{
*E-mail: yanzh@ncu.edu.cn

Received May 5, 2012; revised June 7, 2012; published online June 21, 2012.

Project supported by the Natural Science Foundation of Jiangxi Province (No. 2009GZH0078).

江西省自然科学基金(No. 2009GZH0078)资助项目.
} 


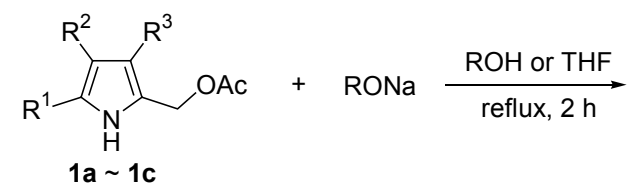<smiles>[R]OCc1[nH]c([14CH3])c([R])c1[R]</smiles>

\section{1 实验部分}

\section{1 主要仪器与试剂}

Bruker 公司 AVANCE $400 \mathrm{MHz}$ 型核磁共振仪(TMS 作内标, $\mathrm{CDCl}_{3}$ 作溶剂); Nicolet 5700 FT-IR 红外光谱仪; Bruker Daltonics Esquire 3000 高分辨质谱仪; Waters ZQ 4000/2695 质谱仪. 柱层析用吸附剂为青岛海洋化工厂 生产的硅胶 H (10 40 $\mu, 200 \sim 300$ 目), 洗脱剂为不同 体积比例的石油醚(沸程为 $60 \sim 90{ }^{\circ} \mathrm{C}$ ) 和乙酸乙酯的混 合溶剂. 所使用的各种醇钠均为现制现用, 所使用的甲 醇、乙醇、异丙醇、正丁醇和苠醇均经镁处理过并常压 蒸馏而得. 无水四氢呋喃(THF) 是将商品化学纯 THF 用 钠条和二苯甲酩回流至蓝色再蒸馏而得. 底物 $1 \mathrm{a} \sim 1 \mathrm{c}$ 是按照文献 $[10 \sim 12]$ 的方法制备的.

\subsection{2-乙酰氧甲基吡咯衍生物和醇钠在醇中的醚化反} 应

在 $25{ }^{\circ} \mathrm{C}$ 和氮气保护下, 将 $2.0 \mathrm{mmol}$ 金属钠加入到 $20 \mathrm{~mL}$ 无水醇中并搅拌直到金属钠完全溶解. 接着往反 应液中加入 $1.0 \mathrm{mmol}$ 底物 $\mathbf{1}$, 并升温到回流状态下继续 反应若干小时. 通过 TLC 来跟踪反应进程. 原料转化完 全后, 将反应液冷却到室温, 加入 $0.1 \mathrm{~mL}$ 乙酸, 旋蒸浓 缩除去挥发性溶剂. 所得残留液用 $20 \mathrm{~mL}$ 乙酸乙酯稀 释, 并用水和饱和食盐水各洗涤一次. 所得油层用无水 硫酸钠干燥, 过滤并浓缩后得到的粗品最后经硅胶柱层 析纯化 [洗脱剂为 $V$ (乙酸乙酯 $): V$ (石油醚 $)=1: 5$ 的混 合溶剂], 得油状物 2 .

\subsection{2-乙酰氧甲基吡咯衍生物和烷氧基钠在四氢呋喃 中的醚化反应}

在 $25{ }^{\circ} \mathrm{C}$ 和氮气保护下, 将 $2.0 \mathrm{mmol}$ 氢化钠(含量为 $60 \%$, 吸附于矿物油中)加入到溶有 2-乙酰氧甲基吡咯 衍生物底物 $1 \mathbf{b}(1.0 \mathrm{mmol})$ 和苠醇 $(1.0 \mathrm{mmol})$ 的 $20 \mathrm{~mL}$ 无 水四氢呋喃溶液中. 接着升温到回流状态下继续反应若 干小时. 通过 TLC 来跟踪反应进程. 原料转化完全后, 将反应液冷却到室温, 加入 $0.1 \mathrm{~mL}$ 乙酸, 旋蒸浓缩除去 挥发性溶剂. 所得残留液用 $20 \mathrm{~mL}$ 乙酸乙酯稀释, 并用 水和饱和食盐水各洗涤一次. 所得油层用无水硫酸钠干
燥, 过滤并浓缩后得到的粗品最后经硅胶柱层析纯化 [洗脱剂为 $V$ (乙酸乙酯 $): V$ (石油醚 $)=1: 5$ 的混合溶剂], 得油状物 $2 \mathbf{f}$.

\section{4 化合物的表征}

2-乙氧甲基-4-硝基吡咯(2a): ${ }^{1} \mathrm{H}$ NMR $\left(\mathrm{CDCl}_{3}, 400\right.$ MHz) $\delta: 9.92$ (br s, 1H, NH), 7.61 (dd, $J=1.6,1.6 \mathrm{~Hz}, 1 \mathrm{H}$, Pyrrole), 6.64 (s, 1H, Pyrrole), 4.44 (s, 2H, CH $\mathbf{H}_{2} \mathrm{OEt}$ ), 3.52 (q, $\left.J=7.2 \mathrm{~Hz}, 2 \mathrm{H}, \mathrm{CH}_{2} \mathrm{OCH}_{2} \mathrm{CH}_{3}\right), 1.20$ (t, $J=7.2 \mathrm{~Hz}, 3 \mathrm{H}$, $\left.\mathrm{CH}_{2} \mathrm{OCH}_{2} \mathrm{CH}_{3}\right) ;{ }^{13} \mathrm{C} \mathrm{NMR}\left(\mathrm{CDCl}_{3}, 75 \mathrm{MHz}\right) \delta$ : 136.91, 129.74, 120.47, 104.13, 65.75, 64.54, 15.03; IR (KBr) $v$ : 3205.43, 3147.55, 2979.48, 2873.63, 1636.64, 1584.64, 1490.30, 1375.75, 1273.06, $1110.77 \mathrm{~cm}^{-1}$; ESI-MS $\mathrm{m} / \mathrm{z}$ : $170\left(\mathrm{M}^{+}\right)$. Anal. calcd for $\mathrm{C}_{7} \mathrm{H}_{10} \mathrm{~N}_{2} \mathrm{O}_{3}$ : C 49.41, H 5.92, N 16.46; found C 49.52, H 5.98, N 16.22.

2-异丙氧甲基-4-硝基吡咯(2b): ${ }^{1} \mathrm{H} \mathrm{NMR}\left(\mathrm{CDCl}_{3}\right.$, $400 \mathrm{MHz}) \delta: 9.27$ (br s, 1H, NH), 7.61 (dd, $J=1.6,1.6 \mathrm{~Hz}$, 1H, Pyrrole), 6.62 (s, 1H, Pyrrole), 4.46 (s, 2H, CH $\left.\mathbf{H}_{2} \mathrm{OPr}-i\right)$, $3.70 \sim 3.67\left(\mathrm{~m}, 1 \mathrm{H}, \mathrm{CH}_{2} \mathrm{OCHMe}\right.$ ), $1.19[\mathrm{~d}, J=6.4 \mathrm{~Hz}$, $\left.6 \mathrm{H}, \mathrm{CH}_{2} \mathrm{OCH}\left(\mathrm{CH}_{3}\right)_{2}\right] ;{ }^{13} \mathrm{C} \mathrm{NMR}\left(\mathrm{CDCl}_{3}, 75 \mathrm{MHz}\right) \delta$ : $137.35,130.28,119.48,103.00,71.50,62.23,22.00$; IR (KBr) v: 3430.00, 3186.85, 3145.24, 3002.64, 1637.11, 1495.37, 1400.22, 1275.11, $1110.13 \mathrm{~cm}^{-1}$; ESI-MS m/z: $184\left(\mathrm{M}^{+}\right)$. Anal. calcd for $\mathrm{C}_{8} \mathrm{H}_{12} \mathrm{~N}_{2} \mathrm{O}_{3}$ : C 52.16, H 6.56, N 15.20; found C 52.42, H 6.60, N 15.30.

2-正丁氧甲基-4-硝基吡咯(2c): ${ }^{1} \mathrm{H} \mathrm{NMR}\left(\mathrm{CDCl}_{3}\right.$, $400 \mathrm{MHz}$ ) $\delta$ : 9.19 (br s, 1H, NH), 7.62 (d, $J=1.6 \mathrm{~Hz}, 1 \mathrm{H}$, Pyrrole), 6.64 (s, 1H, Pyrrole), 4.44 (s, 2H, CH $\left.\mathbf{H}_{2} \mathrm{OBu}\right), 3.46$ $\left(\mathrm{t}, J=6.8 \mathrm{~Hz}, 2 \mathrm{H}, \mathrm{CH}_{2} \mathrm{OCH}_{2} \mathrm{Pr}-n\right), 1.60 \sim 1.56(\mathrm{~m}, 2 \mathrm{H}$, $\left.\mathrm{OCH}_{2} \mathrm{CH}_{2} \mathrm{Et}\right), \quad 1.37 \sim 1.35\left(\mathrm{~m}, 2 \mathrm{H}, \mathrm{OCH}_{2} \mathrm{CH}_{2} \mathrm{CH}_{2} \mathrm{CH}_{3}\right)$, $0.90\left(\mathrm{t}, J=7.4 \mathrm{~Hz}, 3 \mathrm{H}, \mathrm{OCH}_{2} \mathrm{CH}_{2} \mathrm{CH}_{2} \mathrm{CH}_{3}\right) ;{ }^{13} \mathrm{C} \mathrm{NMR}$ $\left(\mathrm{CDCl}_{3}, 75 \mathrm{MHz}\right) \delta: 137.39,129.90,119.62,103.53$, 70.35, 64.82, 31.59, 19.26, 13.83; IR (KBr) v: 3213.81, 3149.61, 3009.49, 2959.42, 2933.77, 2869.52, 1584.15, 1492.36, 1380.16, 1274.58, $1109.90 \mathrm{~cm}^{-1}$; ESI-MS m/z: $198\left(\mathrm{M}^{+}\right)$; HRMS calcd for $\mathrm{C}_{9} \mathrm{H}_{14} \mathrm{~N}_{2} \mathrm{O}_{3}$ 198.2035, found 198.2038

3-甲基-5-乙氧甲基吡咯-2-羧酸乙酯(2d): ${ }^{1} \mathrm{H}$ NMR $\left(\mathrm{CDCl}_{3}, 400 \mathrm{MHz}\right) \delta: 9.25$ (br s, $\left.1 \mathrm{H}, \mathrm{NH}\right), 5.90$ (d, $J=2.6$ $\mathrm{Hz}, 1 \mathrm{H}$, Pyrrole), 4.34 (s, 2H, $\mathrm{CH}_{2} \mathrm{OEt}$ ), 4.23 (q, $J=7.2$ $\mathrm{Hz}, 2 \mathrm{H}, \mathrm{COOCH}_{2} \mathrm{CH}_{3}$ ), 3.42 (q, $J=6.9 \mathrm{~Hz}, 2 \mathrm{H}, \mathrm{CH}_{2} \mathrm{O}-$ $\mathrm{CH}_{2} \mathrm{CH}_{3}$ ), 2.24 (s, 3H, Pyrrole-CH $\left.\mathbf{H}_{3}\right), 1.27$ (t, $J=7.1 \mathrm{~Hz}$, $3 \mathrm{H}, \mathrm{COOCH}_{2} \mathrm{CH}_{3}$ ), 1.12 (t, $J=7.0 \mathrm{~Hz}, 3 \mathrm{H}, \mathrm{RCH}_{2} \mathrm{OCH}_{2}-$ $\left.\mathrm{CH}_{3}\right) ;{ }^{13} \mathrm{C} \mathrm{NMR}\left(\mathrm{CDCl}_{3}, 75 \mathrm{MHz}\right) \delta: 161.64,132.71$, $128.11,119.06,111.48,65.60,64.93,59.75,14.99,14.40$, 12.67; IR (KBr) v: 3434.60, 3143.03, 1613.24, 1400.09 
$\mathrm{cm}^{-1}$; ESI-MS m/z: $211\left(\mathrm{M}^{+}\right)$; HRMS calcd for $\mathrm{C}_{11} \mathrm{H}_{17} \mathrm{NO}_{3}$ 211.3017, found 211.3015.

3-甲基-5-甲氧甲基吡咯-2-羧酸甲酯(2e): ${ }^{1} \mathrm{H}$ NMR $\left(\mathrm{CDCl}_{3}, 400 \mathrm{MHz}\right) \delta: 9.58(\mathrm{br} \mathrm{s}, 1 \mathrm{H}, \mathrm{NH}), 5.97$ (d, $J=2.5$ $\mathrm{Hz}, 1 \mathrm{H}$, Pyrrole), 4.37 (s, 2H, $\mathrm{CH}_{2} \mathrm{OMe}$ ), 3.82 (s, $3 \mathrm{H}$, $\mathrm{COOCH}_{3}$ ), 3.32 (s, $3 \mathrm{H}, \mathrm{CH}_{2} \mathrm{OCH}_{3}$ ), 2.30 (s, 3H, Pyrrole$\left.\mathrm{CH}_{3}\right) ;{ }^{13} \mathrm{C}$ NMR $\left(\mathrm{CDCl}_{3}, 75 \mathrm{MHz}\right) \delta: 161.97,132.47$, 128.42, 119.05, 67.00, 58.00, 51.05, 12.69; IR (KBr) v: $3428.59,3130.97,1639.53,1399.32 \mathrm{~cm}^{-1}$; ESI-MS $m / z$ : $184(\mathrm{M}+1)^{+}$. Anal. calcd for $\mathrm{C}_{9} \mathrm{H}_{13} \mathrm{NO}_{3} \bullet 0.3 \mathrm{H}_{2} \mathrm{O}: \mathrm{C}$ 57.31, H 7.26, N 7.42; found C 57.55, H 7.34, N 7.69.

3-甲基-5-芐氧甲基吡咯-2-羧酸乙酯(2f): ${ }^{1} \mathrm{H}$ NMR $\left(\mathrm{CDCl}_{3}, 400 \mathrm{MHz}\right) \delta: 8.96($ br s, $1 \mathrm{H}, \mathrm{NH}), 7.38 \sim 7.31(\mathrm{~m}$, $5 \mathrm{H}, \mathrm{ArH}), 5.99$ (s, 1H, Pyrrole), 4.52 (s, 2H, $\mathrm{CH}_{2} \mathrm{OBn}$ ), $4.47\left(\mathrm{~s}, 2 \mathrm{H}, \mathrm{CH}_{2} \mathrm{OCH_{2 }} \mathrm{Ph}\right), 4.31(\mathrm{q}, J=7.0 \mathrm{~Hz}, 2 \mathrm{H}$, $\mathrm{COOCH}_{2} \mathrm{CH}_{3}$ ), 2.33 (s, 3H, Pyrrole- $\left.\mathrm{CH} \mathbf{H}_{3}\right), 1.35$ (t, $J=7.0$ $\left.\mathrm{Hz}, 3 \mathrm{H}, \mathrm{COOCH}_{2} \mathrm{CH}_{3}\right) ;{ }^{13} \mathrm{C}$ NMR $\left(\mathrm{CDCl}_{3}, 75 \mathrm{MHz}\right) \delta$ : $160.50,136.65,131.21,127.49,127.26,126.92,126.88$, $118.33,110.77,71.14,63.50,58.87,13.52,11.70$; IR (KBr) v: 3442.47, 3132.47, 1664.04, $1399.57 \mathrm{~cm}^{-1}$; ESI-MS $m / z: 274[\mathrm{M}+1]^{+}, 296[\mathrm{M}+\mathrm{Na}]^{+}$; HRMS calcd for $\mathrm{C}_{16} \mathrm{H}_{19} \mathrm{NO}_{3} 273.4063$, found 273.4062 .

4-苯基-5-甲氧甲基吡略-2-羧酸甲酯(2g): ${ }^{1} \mathrm{H}$ NMR $\left(\mathrm{CDCl}_{3}, 400 \mathrm{MHz}\right) \delta: 9.37$ (br s, $\left.1 \mathrm{H}, \mathrm{NH}\right), 7.41 \sim 7.35(\mathrm{~m}$, $4 \mathrm{H}, \mathrm{ArH}), 7.28 \sim 7.25(\mathrm{~m}, 1 \mathrm{H}, \mathrm{ArH}), 7.04(\mathrm{~d}, J=2.8 \mathrm{~Hz}$, $1 \mathrm{H}$, Pyrrole), 4.58 (s, 2H, $\mathrm{CH}_{2} \mathrm{OMe}$ ), 3.88 (s, 3H, COO$\mathrm{CH}_{3}$ ), 3.32 (s, $\left.3 \mathrm{H}, \mathrm{CH}_{2} \mathrm{OCH}_{3}\right) ;{ }^{13} \mathrm{C}$ NMR $\left(\mathrm{CDCl}_{3}, 75\right.$ MHz) $\delta: 161.40,134.92,130.31,128.61,127.78,126.36$, 124.65, 121.71, 114.92, 66.31, 58.49, 51.51; IR (KBr) $v$ : $3442.24,3133.31,1684.45,1640.41,1399.68 \mathrm{~cm}^{-1}$; ESIMS $m / z$ : $245\left(\mathrm{M}^{+}\right)$. Anal. calcd for $\mathrm{C}_{14} \mathrm{H}_{15} \mathrm{NO}_{3}: \mathrm{C} 68.55, \mathrm{H}$ 6.16, N 5.71; found C 68.76, H 6.18, N 6.08.

\section{2 结果与讨论}

\section{1 反应条件的优化}

需要说明的是, 2-乙酰氧甲基吡咯和吡咯环上没有 吸电子基团取代的 2-乙酰氧甲基吡咯衍生物都是不稳 定的物质, 因此, 为方便起见, 我们使用了三个稳定的 吡咯环上含有吸电子基团的 2-乙酰氧甲基吡咯衍生物 $1 \mathrm{a}, 1 \mathrm{~b}$ 和 $1 \mathrm{c}$ 作为底物来考察它们和醇钠的醚化反应.

我们首先以底物 $1 \mathrm{a}$ 与乙醇钠在乙醇中的反应为例, 对反应条件进行了优化, 见 Eq. 2. 这里考察的反应条件 包括反应温度和乙醇钠的摩尔数. 乙醇钠是现制现用 的，是通过钠块在无水乙醇中于室温下搅拌反应直到钠 块消失而制得. 固定乙醇钠的加入量为 2.0 equiv. 考察
了温度对该反应的影响. 实验结果见表 1.

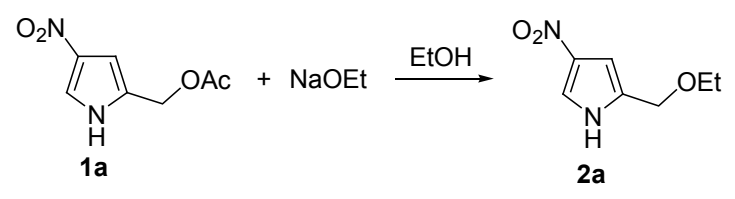

表 1 温度对 $1 \mathrm{a}$ 和乙醇钠的醚化反应的影响

Table 1 Effects of temperature on etherification of 1a with $\mathrm{NaOEt}$

\begin{tabular}{cccc}
\hline Entry & Temperature $/{ }^{\circ} \mathrm{C}$ & Time $/ \mathrm{h}$ & Isolated yield $/ \%$ \\
\hline 1 & 25 & 2 & 0 \\
2 & 45 & 2 & 20 \\
3 & 65 & 2 & 60 \\
4 & 80 & 2 & 92 \\
\hline
\end{tabular}

由表 1 可见, 温度对该醚化反应影响很大. 在室温 下，原料几乎无变化，没有任何醚化产物 2a 生成. 而在 较高温度时, 醚化产物 2a 的量在慢慢增加. 当在回流温 度下反应 $2 \mathrm{~h}$ 后, 原料几乎完全转化成了所需要的产物 2a. 值得一提的是，在室温或加热回流状态下，在反应 体系中我们始终没有发现原料 1a 的水解产物 2-羟甲基3-硝基吡咯的生成, 此外, 对照实验还发现, 纯净的 2羟甲基-3-硝基吡咯和乙醇钠在乙醇中加热回流时，没 有任何反应发生. 另外我们还发现，反应时间对醚化反 应也有一定的影响，在回流条件下反应时间为 $1 \mathrm{~h}$ 时， 原料转化不完全, 延长时间到 $2 \mathrm{~h}$ 时, 原料才转化完全, 继续延长反应时间到 $5 \mathrm{~h}$ 时, 反应情况没有变化. 因此 对 $1 \mathrm{a}$ 和乙醇钠的反应，反应时间以 $2 \mathrm{~h}$ 为好. 接下来, 固定反应温度在 $80{ }^{\circ} \mathrm{C}$ 和反应时间为 $2 \mathrm{~h}$, 我们考察了乙 醇钠的加入量对该醚化反应的影响. 实验结果见表 2 . 从表 2 可见，当 $\mathrm{NaOEt}$ 的使用量为 0.05 equiv. 时，几乎 不发生醚化反应. 当加入量为 0.5 equiv. 时, 也只生成了 少量的醚化产物. 当加入量为 1.0 equiv. 时, 原料即大部 分转化成了醚化产物. 从表 2 的实验结果可见, 当加入 量为 2.0 equiv.时, 醚化产物得率最高.

表 2 乙醇钠的加入量对 1a 的醚化反应的影响 Table 2 Effects of molar equivalent of NaOEt on etherification of $1 \mathrm{a}$

\begin{tabular}{ccc}
\hline Entry & NaOEt/equiv. & Isolated yield/\% \\
\hline 1 & 0.05 & 0 \\
2 & 0.5 & 15 \\
3 & 1.0 & 88 \\
4 & 2.0 & 92 \\
5 & 3.0 & 90 \\
6 & 4.0 & 87 \\
\hline
\end{tabular}

\section{2 不同底物的醚化反应}

为了继续探讨 2-乙酰氧甲基吡咯衍生物和醇钠的 醚化反应的适用范围和反应情况，我们以 $\mathbf{1 a}, \mathbf{1 b}$ 和 $\mathbf{1 c}$ 
为原料底物, 考察了它们与其它一些醇钠, 如甲醇钠、 异丙醇钠、正丁醇钠、叔丁醇钠和苯甲醇钠的醚化反应， 实验结果见表 3.

表 $31 \mathrm{a}, 1 \mathrm{~b}$ 和 $1 \mathrm{c}$ 与醇钠的醚化反应 ${ }^{a}$

Table 3 Etherification of $\mathbf{1 a}, \mathbf{1 b}$ and $\mathbf{1 c}$ with sodium alkoxide

\begin{tabular}{|c|c|c|c|}
\hline Entry & Substrate & $\begin{array}{l}\text { Sodium } \\
\text { alkoxide }\end{array}$ & $\begin{array}{c}\text { Product } \\
\text { (isolated yield) }\end{array}$ \\
\hline
\end{tabular}

1<smiles>CC(=O)OCc1cc([N+](=O)[O-])c[nH]1</smiles>

1a

$\mathrm{NaOPr}-i$

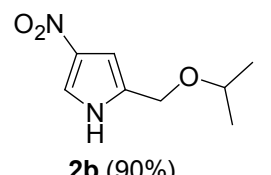

3

1a

$1 \mathbf{a}$<smiles>CC(=O)OCc1cc(C)c(C(=O)O[Na])[nH]1</smiles>

6

1b

$1 \mathrm{~b}$

8<smiles>CC(=O)OCc1cc(-c2ccccc2)c(C(C)=O)[nH]1</smiles>

$\mathrm{NaOEt}$<smiles>CCOCc1cc([N+](=O)[O-])c[nH]1</smiles>

2a (92\%)
$\mathrm{NaOBu}-n$

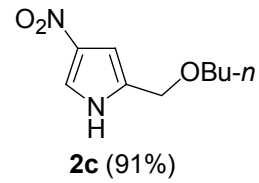

$\mathrm{NaOBu}-t$

$\mathrm{NaOEt}$<smiles>CCOCc1cc(C)c(C(=O)OCC)[nH]1</smiles>

$\mathrm{NaOMe}$<smiles>COCc1cc(C)c(C(=O)O[Na])[nH]1</smiles>

$\mathrm{NaOMe}$<smiles>CCOC(=O)c1[nH]c(COCc2ccccc2)cc1C</smiles>

${ }^{a}$ All the reactions were run at reflux; $1: \mathrm{NaOR}=1: 2$ (molar ratio); the reaction time is $2 \mathrm{~h} ;{ }^{b}$ The reaction of $\mathbf{1 b}$ with $\mathrm{NaOBn}$ was run at reflux in THF, and $\mathrm{NaOBn}$ was obtained through reaction of $\mathrm{NaH}$ with $\mathrm{BnOH}$; and reaction time is $8 \mathrm{~h}$.

从表 3 可见, 叔丁醇钠(表 3, Entry 4)和 $\mathbf{1 a}$ 的反应没 有生成相应的醚化产物, 而只是生成了未经鉴定的其它 复杂的产物, 这可能是由于叔丁醇钠具有较大的位阻的
原因. 其它所考察过的甲醇钠、乙醇钠、异丙醇钠、正 丁醇钠和茮醇钠都能很顺利地和 2-乙酰氧甲基吡咯衍 生物发生醚化反应并以极好的得率生成了相应的醚化 产物.

由于苠醇和金属钠反应很慢, 因此表 3 中 Entry 7 的反应是在无水 THF 中进行的, 苠醇钠是经由氢化钠 和苠醇反应而得. 从表 3 中 Entry 7 的实验结果可见，延 长反应时间到 $8 \mathrm{~h}$ 后，该醚化反应也能很顺利地进行， 产物得率是 $85 \%$. 由于其它的醇钠可以很方便地通过金 属钠和相应的小分子醇反应而得, 因此表 3 中除 Entry 7 以外其它的反应都是在相应的醇中于回流状态下进行 的. 表 3 中 Entry 6 的实验除了发生醚化反应外，还发生 了酯交换反应. 表 3 的结果还表明, 吡咯环上其它取代 基对醚化反应几乎没有影响.

\section{3 反应机理探讨}

在前文 ${ }^{[9]}$ 中, 我们提出了“氮杂富勒烯(Azafulvene)” 中间体的机理可以很好地解释吡咯-2-甲醛缩醛衍生物 和醇钠在醇中的缩醛交换反应。参考这一实验结果，在 本文中, 我们认为 2-乙酰氧甲基吡咯衍生物和醇钠的醚 化反应也是通过氮杂富勒烯中间体进行的. 见图 1 (以 $1 \mathrm{a}$ 为例).

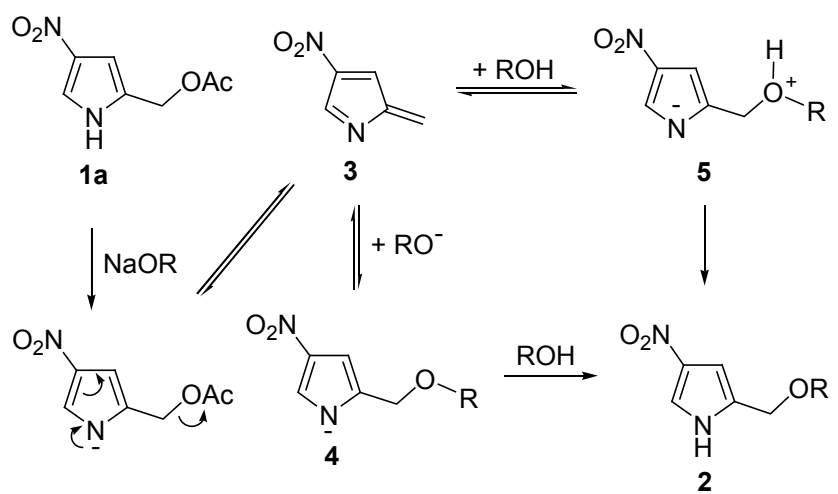

图 1 醚化反应的可能机理

Figure 1 Proposed mechanism of etherification

由于醇钠是一种强碱，因此 $1 \mathbf{a}$ 在醇钠作用下首先 生成氮负离子并通过共振作用转化为不稳定和高度活 泼的氮杂富勒烯中间体 3,3 接着受到烷氧负离子或醇的 进攻而生成中间体 4 或 5 并进一步转化到产物 2. 按照 这一机理解释，若吡咯环的氮原子上有烷基取代时，在 强碱介质中应该不会生成活泼的中间体 3 , 因此醚化反 应就不会发生. 为了验证这一假设, 我们制备了 $N^{1}$-甲 基-3-硝基-2-乙酰氧甲基吡咯并考察了该化合物在甲醇 中于回流状态下和甲醇钠的反应，实验结果证实了我们 的设想，反应确实是没有生成相应的醚化产物，而可能 是首先生成了水解产物, 在高温下, 该水解产物不稳定 
进而转化成了复杂的其它产物. 同样, 由于苯环中不存 在和 3 相似的中间体, 因此乙酸苠酯在甲醇中于回流状 态下和甲醇钠的反应也不会形成醚化产物甲氧甲基苯, 而只有水解产物苯甲醇生成, 这一点也被我们的实验所 证实.

总之, 针对 $N^{1}$ 未取代的 2-乙酰氧甲基吡咯衍生物 在强碱作用下易于生成高度活泼的氮杂富勒烯中间体 $\mathbf{3}$ 的特性，我们考察了该类化合物和醇钠的梄化反应，结 果以几乎定量的得率生成了相应的 2-烷氧甲基吡咯衍 生物. 吡咯环上其它取代基对醚化反应没有影响. 该类 醚化反应尤其适合于制备对酸敏感的 2-烷氧甲基吡咯 衍生物. 由于 2-乙酰氧甲基吡咯衍生物比 2-着圣甲基吡咯 衍生物更稳定, 因此本工作为从 2-乙酰氧甲基吡咯衍生 物出发制备 2-烷氧甲基吡咯衍生物提供了一种很有效 的方法.

\section{References}

[1] Kinoshita, H.; Tanaka, S.; Nishimori, N.; Dejima, H.; Inomata, K. Bull. Chem. Soc. Jpn. 1992, 65, 2660.

[2] Kinoshita, H.; Tanaka, S.; Inomata, K. Chem. Lett. 1989, 1107.

[3] Kijewska, K.; Kisiel, A.; Michaiska, A. Chem. Eur. J. 2012, 18, 310.

[4] Modak, A.; Nandi, M.; Mondal, J.; Bhaumik, A. Chem. Commun. 2012, 48, 248.

[5] Coelho, P. J.; Castro, M. C. R.; Fonseca, A. M. C.; Raposo, M. M. M. Dyes Pigm. 2012, 92, 745.

[6] Padwa, A.; Fryxell, G. E.; Gasdaska, J. R.; Venkatramanan, M. K.; Wong, G. S. K. J. Org. Chem. 1989, 54, 644.

[7] Silva, M. R.; Sobral A. J.; Silva, J. A. J. Chem. Crystallogr. 2007, $37,695$.

[8] Dinsmore, A.; Mandy, K.; Michael, J. P. Org. Biomol. Chem. 2006, 4(6), 1032.

[9] Yan, Z.; Kang, R.; Liu, Y.; Lin, S. Chin. Chem. Lett. 2012, 23, 33

[10] Montforts, F. P.; Schwartz, U. M. Liebigs Ann. Chem. 1985, 2301.

[11] Smith, J. A.; Ng, S.; White, J. Org. Biomol. Chem. 2006, 4, 2477.

[12] Silverstein, R. M.; Ryskiewicz, E. E. J. Org. Chem. 1955, 20, 668.

(Li, L.; Fan, Y.) 